\title{
InFoamed Matter
}

\section{Robotic production and assembly of foam-injected structures}

\author{
Shota Tsikoliyal ${ }^{1}$ David Kovařik ${ }^{2}$, Imro Vasko ${ }^{3}$, Petra Garajová ${ }^{4}$, \\ Adam Varga ${ }^{5}$, Marketa Osifová ${ }^{6}$ \\ 1,2,3,4,5,6 Academy of Arts Architecture and Design in Prague \\ 1,3\{shota.tsikoliya|imro.vasko\}@vsup.cz² kovarik.d@gmail.com
}

\begin{abstract}
Project InFoamed Matter works with foam and explores the internal logic of the material and develops a construction system based on fluidity and expansion. The basic unit of the system consists of two elements, that continuously exchange their roles in the construction process - the frame (controlling element made of paper or, in later development, from glass or carbon fiber cured in epoxy resin) and the expander (filling element consisting of $2 k$ polyurethane foam). The expander fills up voids within the frame. While initially only the frame plays crucial structural role within a system, the expander being a filling element, eventually the hardening process switches the roles, hardened expander being the structural core and the frame being a form-defining tool. In later development, fiber frame creates a composite together with hardened expander, being able to resist both tension and compression forces. Project further proposes computational model, which generates positions and orientations for placing further components as well as a robotic fiber laying, assembly and injection system, which leads to novel automated construction system based on material behavior.
\end{abstract}

Keywords: robotic fabrication, foam, materiality, robotic assembly

Project InFoamed Matter is inspired by a notion, that in nature material is heterogeneously distributed to fit its structural and/or environmental performance, and that such structural heterogeneity (also termed difference, or differentiation) is made possible due to the integration between form, structure and material (Oxman 2010, p.31). 3D printing revolutionized the materialization of digital concepts and brought the internal logic of the simulation systems into physical reality. It entered the market as a method for producing prototype components, then for produc- ing tooling and molds and, most recently, for 'enduse' parts. This evolution reflects improved, selectively curable materials that have extended their performance to match standard engineering polymers and metal alloys. Additive manufacturing has penetrated high-value, short-run and 'custom' products due to increased complexity, design flexibility, material utilization and increased functionality, compared to the traditional engineering processes of forming or machining components. (Soar, Andreen, 2012, p. $128)$, however it rarely truly pursues the internal logic 
of material behavior. The necessity of deconstruction of any material into its liquid or powder forms brings in a potential in terms of available geometry, but it also means, that various properties of the material related to its inner heterogenic structure are lost. This leads to the dematerialization of architecture a lost connection between materiality and geometry. The project InFoamed Matter attempts to reverse that process. If follows the material logic and directly explores the behavior of materials. Materials are not seen as a matter that gives shape to abstract, immaterial geometries, but rather as a generative element that produces shapes, properties and relations.

Project InFoamed Matter aims to develop new generations of geometry-based material composites, a strategy that integrates material performance and digital technologies, and investigates synergies between them. Material performance is a sum of all the diverse present properties of certain material. These include among others insulation, acoustic, chemical or thermal properties, but also the geometrical ones in micro and macro scale as well as the general behavior of the material. The digital technologies are geometrical form-generating or form-optimizing tools commonly used in contemporary architecture, but also robotic production or 3D printing. The present research development in architecture tends to favor either one or the other attitude. The properties of single materials are often put together to form a "properties component" such as sandwich wall system. On the other hand, the robotic production or $3 \mathrm{D}$ printing is vastly used to transform the digital design into real world without considering material performance. The new composites connect the outputs of both tendencies. The geometrical designed digital forms and assemblies are specially shaped and optimized to support material performance. On the other hand, the material and its performance is seen as an integrated part of the generating algorithm, an analogue plug-in.

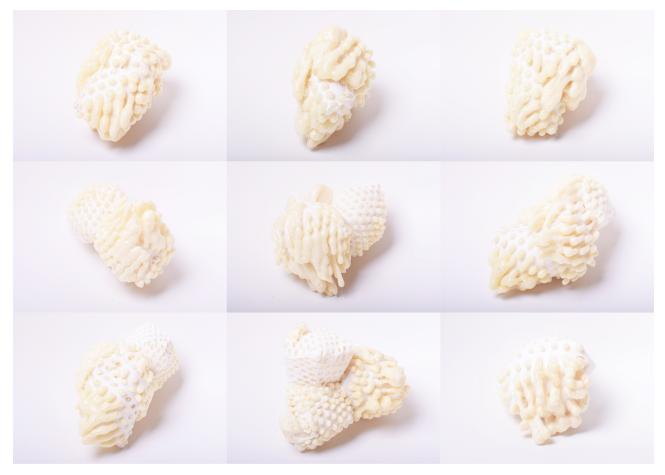

The project is focused on working with $2 \mathrm{k}$ expanding polyurethane foam, material consisting of two stable liquid chemical components. By mixing the components together, the initial properties of the liquids change. The mixture rapidly expands, losing its fluidity, multiplying its volume and finally hardening. Observation of the process offers several properties through which the expansion could possibly by controlled.Mixing up both components initiates the process of expansion. Different ratios of the components result in different types of foam and different properties of the material. For the purpose of optimal expansion, one stable ratio was selected. Once initiated by mixing up two single components, the material massively expands its volume and hardens after short period of time. Within this period, the foaming matter can be controlled, influenced and redirected. The process of expansion is seen as a pre-programmed behavior that can be actively influenced and used for architectural solutions. The basic unit of the proposed system consists of two elements constantly changing their roles in the construction process - the expander and the frame. The expander corresponds with the material ( $2 \mathrm{k}$ polyurethane foam) and its natural behavior. The frame acts as a controlling element of the material - takes over the role of the environment the material is placed in. Depending on the situation, the system is either more opened (material-oriented) or more controlled (environment oriented). The proposed construction process oscillates between these two poles. Under stable conditions, the expander
Figure 1 Foam expansion within variable frame arangements - tests 
Figure 2

Injection, expansion and assembly sequence

Figure 3

Computation,

fabrication, material and sensing

systems performs every time the same, but the frame varies depending on local requirements producing different results. Through different types of frames different performance of the expander can be achieved (fig. 1). The expansion can be slowed-down, redirected or speed up. This offer a great potential not only for optimizing structures, but also for achieving specific architectonic solutions.

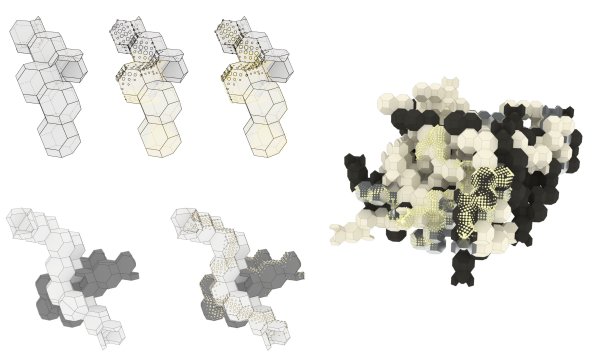

The assembly logic of the prototype is driven by the necessity to create structural skeleton out of the hardened foam within the frames (fig.2). The bottomup process, based on variation of positions of the module, evaluated by their ability to ensure the distribution of the material deep inside the structure as well as the ability to create a foam connection between the single modules, uses various behaviors to create basic building elements, such as "wall", "cantilever" or "arch". Globally defined constraints ensure that the process reaches the pre-designed overall shape (or not trespasses given spatial constraints), while its particular geometry is defined by the necessity of foam distribution in a way to ensure stability of the structure at each stage of construction. An integral part of the assembly is a computational model, a multi-agent system in a three-dimensional voxel grid. The core of a multi-agent model is three local behaviors. The model evaluates local conditions, such as the location and state of a particular voxel, the state of adjacent voxels, and as a result of this evaluation, chooses one of the behaviors. As opposed to instruc- tions, behaviors are nonlinear and their application depends on many factors. While individual behaviors are relatively simple, their applications produce qualities that are visible at a macroscopic level. Another important part of the design of the computational model was the constraints, especially the material hardening and assembly constraints. At each step of updating the agent position, the system has checked whether the assembly is possible, whether it is within the overall limits of the structure, and whether the structure will be stable considering the current state of hardening in different voxels. Segment orientation also affected the feasibility of the step, as it would directly influence the stream of not yet hardened foam. As a result, all of the described above inputs affect the generative behavior of the computational model (fig. 3). The evaluation algorithm was included in the computational system together with a set of evaluation criteria. These include the structural stability of the system and the time-efficiency of the assembly process. This has made it possible to exclude the least promising developmental variants from further evaluation.

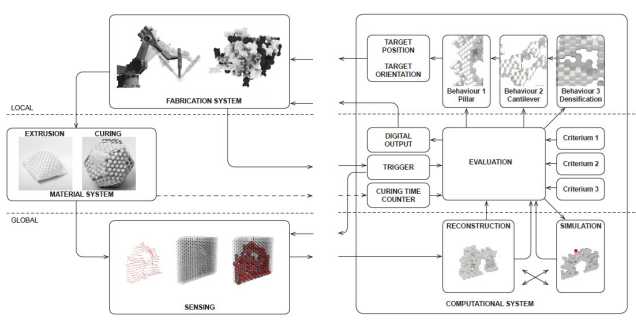

The potential of automated assembly is given by the discrete character of the components. Within the project authors work with limited number of segments and limited number of connection types, bringing properties of truly digital materiality of the project (Retsin, 2016, p. 145). The discrete "Lego" character of the voxels is augmented by the analogue and non-repetitive material behavior of the foam, which distinguished the aesthetics of the de- 
sign from several recent researches within the field of discrete architecture. While in the initial tests the assembly and injection process were done manually, in the later stages the research concentrates on further development of fully automated construction. Robotic arm uses gripper to correctly position and orient each component, while inject the foam is injected inside of the component. After the hardening of the foam, the component becomes an integral part of the existing structure and the robotic arm proceeds to the next step.

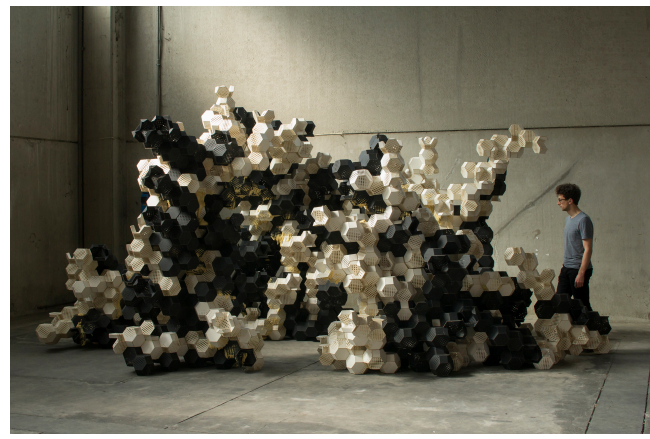

In later development project has worked with a spherical frame constructed from robotically laid glass or carbon fiber cured in epoxid resin on a pneumatic formwork (fig.5). Such frame had the advantages of being able to carry higher structural loads, particularly in compression, as well as providing far richer variety of assembly positions. A single frame has included circular openings, which fitted other spherical frames, as well as provided a possibility for the expander to transfer form one frame to another, while liquid. Computational system consists of two parts: 1. Agent-based system is used to generate the appropriate aggregation of the components. The system evaluates the performance of the given aggregation in terms of structural stability and several custom-given criteria, such as transparency, roughness, surface to volume ratio, volume of the enclosed space etc. Agents are aggregated along the desired surfaces, curves or masses, while keeping the given minimal distances between each other. The closest neighboring agents create a proximity mesh, which in turn works as a basic geometry for generation of the frames sizes, positions and orientations. 2. Another agent-based system is used to generate appropriate fiber laying pattern for each frame, in a way to accommodate connection openings, transfer the loads and keep the required level of perforation for optimal curing of the expander. Agents travel on the surface of the sphere, leave a fiber path behind them, minimize the curvature of the pattern, avoid openings and reinforce the edges around them as well as the areas of particular compression loads. Following that, an assembly sequence is generated, similar to one used in earlier development and described above. A transition of soft pneumatic formwork into a rigid carbon fiber reinforced shell was previously successfully tested in ICD/ITKE research pavilion 2013 by the researchers from Stuttgart University. Several methods developed in the research are successfully implemented in the process, including robotic mapping of the formwork surface in advance to the fiber laying sequence as well as agent-based generation of the fiber pattern. (Moritz Doerstelmann, Jan Knippers, Valentin Koslowski, Achim Menges, Marshall Prado, Gundula Schieber and Lauren Vasey, 2015, p. 60-65).
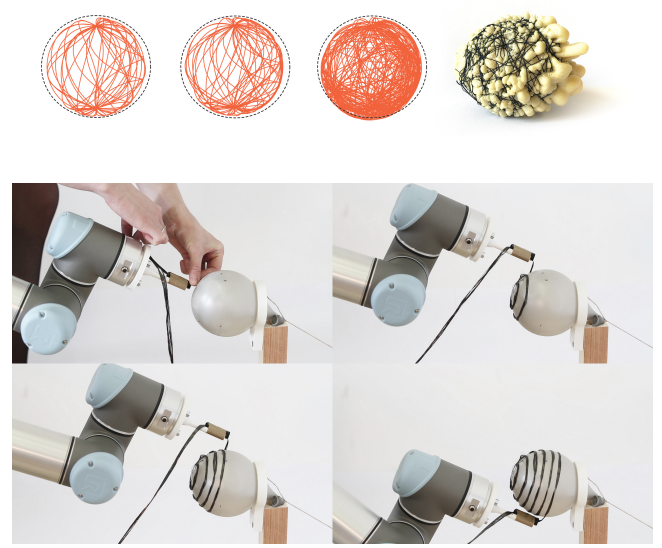

Figure 4 InFoamed Matter final installation

Figure 5

Fiber laying path generation and fabrication

Figure 6 Robotic laying of fiber 
In this stage of the project, the role of the robotic arm is focused on both creating the frame through laying carbon or glass fiber over spherical formwork using custom-made effector (fig.6), as well as its correct positioning during the assembly (fig.7). The latter is particularly important considering that spherical frames don't have limited amount of connections, unlike their earlier development stages made out of paper. The paths for the robotic arm are generated in advance (using plugin ROBOTS and within Rhinoceros/GH environment) and are manually actualized based on the actual positions of the frames after foam expansion.

Figure 7

Robotic assembly process bly and "inFoaming" injection process, which can lead to the emergent architecture, defined by complex material behaviors. The project is the result of a gap between materiality and geometry, present in almost all the major fields of building industry. Although pioneering projects in that field have been constructed, most of architecture can't profit from the knowledge acquired from the digital design research of the last twenty years, mainly due to the lack of information or missing affordable technology. This gap originates in the separation of design and production in architecture and can be tracked back to the renaissance establishment of architecture as an authoritarian work (Carpo, 2011). In comparison, project methods originate in fundamental experimentation with materials. At the beginning, there is no design intention in any experiment. Materials are seen as a pre-programmed matter which behavior can be tracked, understood and described in the similar way the emergence theory suggests. The actual design methods develop from deep understanding of material's behavior and attempts to influence and control it. This approach leads to the relation of a material and the environment the material is placed in. The further phases of the project will focus on improving the material performance and reducing the environment to its most necessary elements. This can potentially lead to assembly of reduced, yet flexible geometrical frameworks imbued with material performance and carry out the idea of digital material. Similar systems can be developed for different materials, with potential of automated or self- assemblies, which is particularly demanded in order to realize the full potential of the bottom-up and collective character of the assembly process ( $\mathrm{Lu}$, 2017, p. 133).

The new generation of geometry-based material composites reveal new possibilities of synergies between digitally generated geometries and materials. Seeing material performance as an analogue plug-in of processing algorithm, the In-Foamed Matter questions the present construction systems in building industry, as well as the production standards. If com- 
pared to commonly used construction systems (like reinforced concrete), the new, process oriented composites offer higher flexibility and introduce digital designing methods in material systems. The digital design process brings in all the potential, but also risks of non standard seriality: loosing the modern specialization in favour of spontaneous adaptivity, acquiring collective intelligence but loosing individuality of a single unit (Carpo, 2017). Architecture emerging from the system resembles naturally organized systems and could possibly be an answer to constructions in non-standard conditions. The robotic production is the first step towards integration of the system into existing construction and production systems.

\section{REFERENCES}

Carpo, M 2011, alphabet and the algorithm, MIT Press, Cambridge, Mass.

Carpo, M 2017, The second digital turn: design beyond intelligence, MIT Press, Cambridge, Mass.

Doerstelmann, M, Knippers, J, Koslowski, V, Menges, A, Prado, M, Schieber, G and Vasey, L 2015, 'ICD/ITKE Research Pavilion 2014-15: Fibre Placement on a Pneumatic Body Based on a Water Spider Web', Architectural Design, 85, pp. 60-65

Lu, A 2017, 'Autonomous Assembly as the Fourth Approach to Genereic Construction', in Tibbits, S (eds) 2017, Autonomous Assembly: Designing for a New Era of Collective Construction, Wiley

Oxman, N 2010, Material-based design computation, Ph.D. Thesis, Massachusetts Institute of Technology. Dept. of Architecture.

Retsin, G 2016 'Assembly and Digital Materials in Architecture', Proceedings of eCAADe 2016

Soar, R and Andreen, D 2012, 'The Role of Additive Manufacturing and Physiomimetic Computational Design for Digital Construction', Architectural Design, 82, pp. 126-128 